\title{
Temperature-Modulated pn-Transition Gas Response of ZnO Nanorod Arrays for Selective Detection of CO
}

\author{
Tingqiang Yang, Wei Jin, Wen Chen \\ State Key Laboratory of Advanced Technology for Materials Synthesis and Processing, School of \\ Materials Science and Engineering, Wuhan University of Technology, Wuhan 430070, P. R. China \\ E-mail: chenw@whut.edu.cn (Wen Chen)
}

\begin{abstract}
It has been problematic to selectively detect $\mathrm{CO}$ by metal oxide semiconductor (MOS) gas sensors, due to gas response to $\mathrm{CO}$ is normally much lower than volatile organic compounds (VOCs). In this work, an abnormal pn-transition gas response of $\mathrm{ZnO}$ nanorod arrays is discovered. At relatively low temperature $(463 \mathrm{~K})$, resistance of $\mathrm{ZnO}$ nanorod arrays increases when $\mathrm{CO}$ is injected into testing chamber, suggesting a "p-type" gas response; while, at relatively high temperature $(643 \mathrm{~K})$, resistance decreases after $\mathrm{CO}$ injection, meaning a usual "n-type" response. Density functional theory (DFT) simulation reveals that the abnormal p-type response at $463 \mathrm{~K}$ is attributed to $\mathrm{CO}$ molecule facilitating the dissociation of absorbed $\mathrm{O}_{2}$ molecule. This peculiarity can be applied to selective detection of $\mathrm{CO}$.
\end{abstract}

Key words: pn-transition, $\mathrm{ZnO}$ nanorod arrays, temperature-modulated, gas sensing, $\mathrm{CO}$

$\mathrm{CO}$ gas is extremely dangerous, not only due to deadly poisonousness, easy combustion and explosion, but also because of colorless and odorless peculiarities. It is definitely necessary to detect $\mathrm{CO}$. Metal oxide semiconductor (MOS) gas sensors have drawn extensive attention in gas sensing domain due to their high gas responses, simple synthesis process and low cost. However, their gas responses to $\mathrm{CO}$ are generally much lower than volatile organic compounds (VOCs), leading to a difficulty in selective detection of $\mathrm{CO}$.

The gas sensing process is generally believed that reducing gases consume absorbed oxygen pieces, thus, give electrons back to surface layers. For n-type MOSs, these processes result in decline of resistance. However, some abnormal phenomena have been reported that resistance of n-type MOSs increases after injection of reducing gas under specific conditions, which is a performance of p-type MOSs [1]. Hence, the abnormal gas response was referred as "p-type" gas response.

In this work, an unprecedented phenomenon is discovered that $\mathrm{ZnO}$ nanorod arrays show $\mathrm{p}$ type gas response to $\mathrm{CO}$ at relatively low temperature and show n-type gas response at higher temperature. The mechanism is revealed by density functional theory (DFT) simulation.
Synthesis of $\mathrm{ZnO}$ nanorod arrays and device fabrication are totally same with our previous works [2]. The gas sensing properties were measured by commercial gas sensing measurement system (WS-30A, Zhengzhou Winsen Technology Corp., China). In the response process, a certain amount of gas was injected into the test chamber, and signals were collected after five minutes to ensure sufficient diffusion of gas molecule. In the recovery process, gas sensor device was exposed to air by opening the chamber for five minutes. After the exposure, the chamber was sealed again, and it was still five minutes before signals acquisition to avoid effects of flow disturbance. Gas response (S) was defined as $R_{\text {air }} / R_{\text {gas }}$, where $R_{\text {air }}$ and $R_{\text {gas }}$ are values of resistance in air and goal gas, respectively.

Fig. 1 shows resistance response curves and gas response curves to $\mathrm{CO}$ at $463 \mathrm{~K}$ and $643 \mathrm{~K}$, respectively. The resistance curve of $463 \mathrm{~K}$ is peculiar. Resistance decreases slightly when $\mathrm{ZnO}$ nanorod arrays are exposed to low concentration of $\mathrm{CO}$, whereas resistance increases if concentration is higher than $50 \mathrm{ppm}$. This results in descent of gas response, shown in Fig. 1c. In contrast, gas sensing properties at $643 \mathrm{~K}$ is normal. Resistance declines obviously upon injection of $\mathrm{CO}$, and gas response rises with enhancement of concentration. 

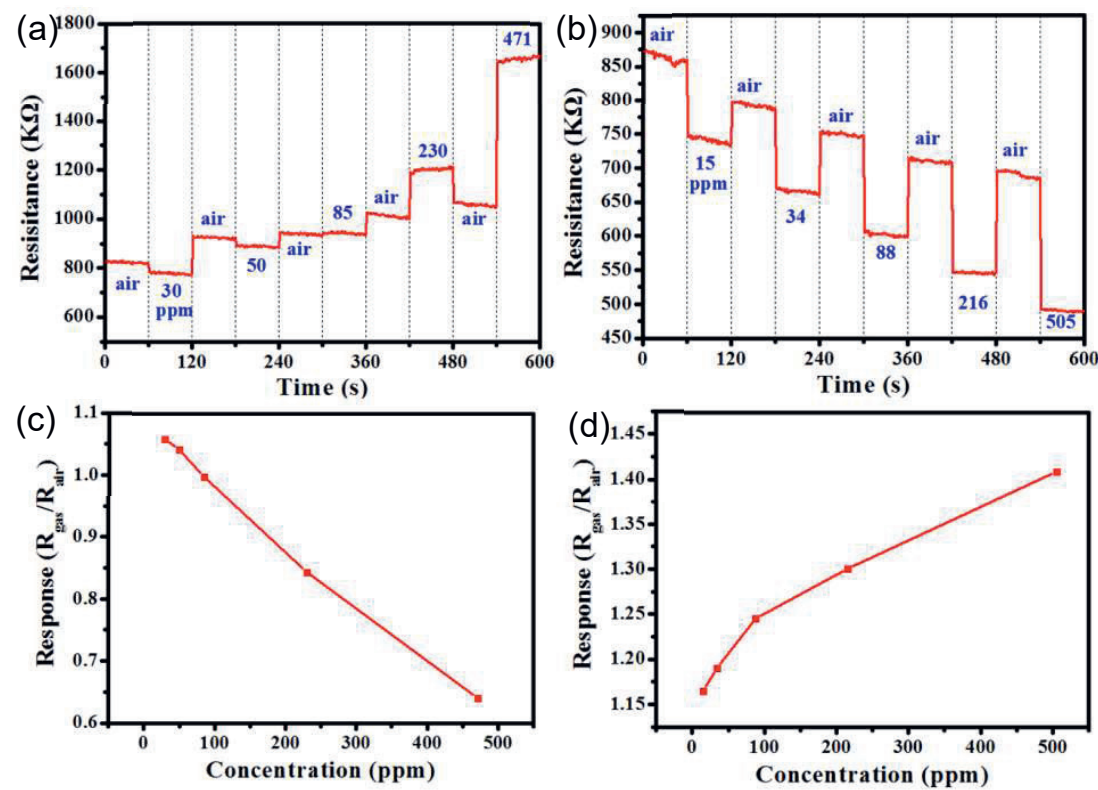

Fig.1. ( $a, b)$ Resistance response curves and (c, d) gas response curves to CO of different concentrations at $463 \mathrm{~K}$ and $643 \mathrm{~K}$, respectively.

Density functional theory (DFT) simulation is utilized to explain the abnormal phenomenon. According to previous research, it is $\mathrm{O}$ adatom that plays role at $643 \mathrm{~K}$. While $463 \mathrm{~K}$ is around the temperature at which absorbed $\mathrm{O}_{2}$ molecule begins to dissociate [2]. Therefore, it is likely that $\mathrm{CO}$ facilitates the dissociation of absorbed $\mathrm{O}_{2}$ molecule. In that case, more electrons will be extracted from $\mathrm{ZnO}$ surface layers, leading to improvement of resistance. Fig.2 illustrates the dissociation process of absorbed $\mathrm{O}_{2}$ molecule on $\mathrm{ZnO}(10 \overline{1} 0)$ with the presence of CO. The activation energy is $342.70 \mathrm{~kJ} / \mathrm{mol}$, which is slightly lower than that $(351.71 \mathrm{~kJ} / \mathrm{mol})$ without CO molecule [2]. This confirms the speculation. The facilitation of $\mathrm{CO}$ on dissociation of $\mathrm{O}_{2}$ molecule is also discovered at $\mathrm{Au}_{\mathrm{x}}$ clusters [3].

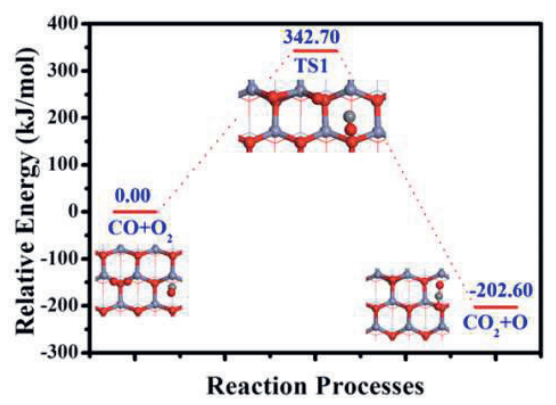

Fig.2. Dissociation process of absorbed $\mathrm{O}_{2}$ molecule with presence of $\mathrm{CO}$ on $\mathrm{ZnO}$ (1010) surface.

This peculiar gas sensing properties can be utilized to detect $\mathrm{CO}$ selectively and quantitatively by modulating working temperature. When the device works at $463 \mathrm{~K}$, it can distinguish $\mathrm{CO}$ from other reducing gases because of abnormal p-type response; when it works at $643 \mathrm{~K}$, concentration of $\mathrm{CO}$ can be determined according to the correlation between gas response and concentration.

In conclusion, it is discovered that $\mathrm{ZnO}$ nanorod arrays show abnormal pn-transition gas response to $\mathrm{CO}$, which is modulated by temperature. The mechanism of p-type response at relatively low temperature is that $\mathrm{CO}$ facilitates the dissociation of absorbed $\mathrm{O}_{2}$ molecule. The peculiarity can be applied to selective detection of $\mathrm{CO}$.

\section{References}

[1] Y. Qin, G. Tao, K. Liu, M. Hu, Vanadium Pentoxide Hierarchical Structure Networks for High Performance Ethanol Gas Sensor with Dual Working Temperature, Sensors and Actuators B: Chemical 190, 141-148 (2014); doi: 10.1016/j.snb.2013.08.061

[2] T. Yang, Y. Liu, W. Jin, Y. Han, S. Yang, W. Chen, Investigation on the Transformation of Absorbed Oxygen at $\mathrm{ZnO}\{10 \overline{1} 0\}$ Surface Based on a Novel Thermal Pulse Method and Density Functional Theory Simulation, ACS Sensors 2, 1051-1059 (2017);

10.1021/acssensors.7b00363

[3] C. Liu, Y. Tan, S. Lin, H. Li, X. Wu, L. Li, Y. Lei, X. Zeng, $\quad \mathrm{CO}$ Self-Promoting Oxidation on Nanosized Gold Clusters: Triangular $\mathrm{Au}_{3}$ Active Site and $\mathrm{CO}$ Induced O-O Scission, Journal of the American Chemical Society 135, 2583-2595 (2013); doi: 10.1021/ja309460v 